\title{
Global Dynamics in Binary Choice Models with Social Influence
}

\section{Gian-Italo Bischi}

Dipartmento di Economia e Metodi Quantitativi (DEMQ), Università di Urbino, Urbino, Italy

\section{Ugo Merlone}

Dipartmento di Statistica e Matematica Applicata, Università di Torino, Torino, Italy

\begin{abstract}
A discrete-time dynamical system is proposed to model a class of binary choice games with externalities as those described by Schelling $(1973,1978)$. In order to analyze some oscillatory time patterns and problems of equilibrium selection that were not considered in the qualitative analysis given by Schelling, we introduce an explicit adjustment mechanism. We perform a global dynamic analysis that allows us to explain the transition toward nonconnected basins of attraction when several coexisting attractors are present. This gives a formal explanation of some overshooting effects in social systems and of the consequent cyclic behaviors qualitatively described in Schelling (1978). Moreover, we show how the occurrence of a global bifurcation may lead to the explanation of situations of path dependence and the creation of thresholds observed in real life situations of collective choices, leading to extreme forms of irreversible departure from an equilibrium and uncertainty about the long run evolution of the some social systems.
\end{abstract}

Keywords: binary games, discrete dynamical systems, global bifurcations, overshooting, social externalities

\section{INTRODUCTION}

In mathematical sociology, according to Sørensen and Sørensen (1977), an important class of models is given by the purposive actor models or rational choice models, as they are more often called. Several sociologists worked within a rational choice framework: Coleman

Address correspondence to Gian-Italo Bischi, Dipartmento di Economia e Metodi Quantitativi (DEMQ), Università di Urbino, via Saffi n. 42, Urbino I-61029, Italy. E-mail: bischi@uniurb.it 
(1973), Granovetter and Soong (1983), and Braun (1995), to cite a few; for a review of rational choice models in sociology the reader may refer to Edling (2002). The application of models where the outcome of an actor's action is also affected by the actions of other actors is growing in sociology. Among the different contributions, the seminal work by Schelling (1973) stands out on its own as it provides a simple model which can qualitatively explain a wealth of everyday life situations. Indeed, the model proposed in Schelling (1973, p. 386), and in the successive versions, is general enough to include several games, such as the well-known $n$-players prisoner's dilemma or the minority games (e.g., Challet and Zhang, 1997). Another important contribution on situations where actors face two alternatives is given in Granovetter (1978); there the approach is based on behavioral thresholds, and the author provides some models that are valuable in helping understand examples where the outcomes are not intuitively consistent with individual preferences.

However, as remarked by Granovetter (1978, p. 1435), in Schelling (1973) the time sequence is not treated because no dynamic models are explicitly given, even if a dynamic adjustment is implicitly assumed in order to both analyze the time evolution of the fraction of agents that make a binary choice and to provide arguments about the existence and stability of equilibrium values. However, this implicit dynamic adjustment fails in describing some important phenomena observed in many real situations, such as oscillations caused by overshooting (or overreaction) of the actors involved in choices repeated over time and problems of equilibrium selection when nonmonotonic payoff curves lead to the presence of several stable equilibria. In the latter case, an explicit formulation of the dynamic process involved is particularly important to describe real situations where extreme forms of path dependence are observed, due to the creation of thresholds that mark irreversible departures from an equilibrium value to reach another distant equilibrium as final outcome (on the importance of thresholds, see also Granovetter, 1978).

We propose an explicit dynamic adjustment that both extends the equilibrium analysis of Schelling (1973) and explores the possible occurrence of nonequilibrium dynamics. In fact, since in Schelling (1973) the time scale is not specified (as noticed also by Granovetter, 1978 , p. 1435), it may be either continuous or discrete. In our approach it seems interesting to investigate what the consequences are on the dynamics depending on the kind of considered time scale. In particular, following Granovetter (1978), we are interested in considering discrete time dynamics since, in social and economic systems, changes over time are usually related to decisions that 
cannot be continuously revised. For example, to quote some of the situations described by Schelling, each year one can decide whether to get a flu vaccination or not; whether to use insecticide or not. Also, in strategic decision making, managers may need to wait for the next board meeting to revise a just-reached decision. Finally, voting for presidential elections and for ballot questions are examples of binary choices where the decisions cannot be continuously revised. Furthermore, in Schelling $(1978$, ch. 3) several other different situations are described and qualitatively analyzed in terms of individuals that make repeated binary choices, that is, a typical discrete time scale (see, e.g., Schelling, 1978, p. 86 or p. 121). As is well known, discrete-time adaptive processes may lead to oscillations, often related to overshooting effects that are quite common in the presence of emotional human decisions. As stressed by Schelling himself (1978), "the phenomenon of overshooting is a familiar one at the level of individual ..." and consequently "numerous social phenomena display cyclical behavior" (p. 86). Also Granovetter (1978) provides several example of binary choices where a discrete time scale seems to be the most appropriate, for example, strikes, votes, and educational attainment. For a discussion, the reader can refer to pages 1423 and 1424 of Granovetter.

In the dynamic model we propose, we assume that individuals make their decisions repeatedly; at each time the strategy each agent chooses depends on the payoffs realized in the previous period, and we use methodologies taken from the qualitative theory of deterministic discrete time dynamical systems in order to study different kinds of long-run evolutions of the collective choices, ranging from convergence to an equilibrium point to persistent oscillations which may be either periodic or chaotic. We will prove that the presence of overshooting and oscillations may substantially modify some of the conclusions on the stability of the equilibria given in Schelling (1973). Moreover, in the case of nonmonotonic payoff curves, when two stable equilibria coexist, as happens in several interesting examples provided by Schelling (1973, p. 414) and Granovetter (1978, p. 1439), the adaptive dynamic process acts as an equilibrium selection device, and the emergence of a particular equilibrium also depends on the history of the collective dynamics. In fact, when several attracting sets coexist, each with its own basin of attraction, the dynamic process becomes path-dependent; that is, which kind of long run dynamics is chosen depends on the initial condition. This rises the question of the delimitation of the basins of attraction; the threshold values that separate the basins of attraction of the different equilibria can be identified as well as their changes as the parameters of the model 
vary. Through a global study of the discrete-time dynamic model proposed in this paper we can show how, in simple situations, a global bifurcation can occur. Such a bifurcation leads to the creation of non connected, that is, separated by multiple thresholds or basins of attraction, which may explain the irreversible departure from an equilibrium we can observe in some real life examples.

Moreover, as usual when discrete time modeling is assumed, periodic or even chaotic time patterns are obtained. In these cases, the delimitation of bounded trapping regions where the asymptotic dynamics of collective behaviors are confined may be an useful information. Indeed, the study of these two kinds of complexity-one related to the complexity of the attracting sets that characterize the long-run time evolution of the dynamic process, the other one related to the complexity of the boundaries which separate the basins when several coexisting attractors are present-requires a global study of the geometric properties dynamic model. The main goal of this article is to study how these complexities arise in a simple explicit dynamical system that models repeated binary choices.

In the spirit of Schelling, we keep the analysis abstract from too many mathematical details. We shall just show how some mathematical methods, which are based on a continuous dialogue between analytic and graphical tools, may be useful to study the global dynamic properties of the model.

The plan is as follows. In Section 2 we present the formal dynamic model; in Section 3 we analyze the cases where the payoff curves have one single intersection; in Section 4 we discuss the case of two intersections and provide an example about the antimicrobial resistance, which illustrates the separation of the basins of attractions Finally, the last section is devoted to concluding remarks.

\section{THE DYNAMIC MODEL}

In order to model how individual choices are influenced by social interactions (social externalities) Schelling (1973, p. 386) proposes two key simplifications. First, each agent (or player) is assumed to have a purely binary choice; second the interaction is impersonal, in the sense that each player's payoff depends only on the number of agents who choose one way or the other and not on their identities. More formally, a population of players is assumed to be engaged in a game where they have to choose between two strategies, for example, $L$ and $R$, respectively. If the set of players is normalized to the interval $[0,1]$ and we denote by the real variable $x \in[0,1]$ the fraction of players that choose strategy $R$, then the payoffs are functions of $x$, 
say $R:[0,1] \rightarrow \mathbb{R}, L:[0,1] \rightarrow \mathbb{R}$, where $R(x)$ and $L(x)$ represent the payoff associated to strategies $R$ and $L$, respectively. Observe that, as we are considering binary choices, when fraction $x$ is playing $R$, then fraction $1-x$ is playing $L$. Obviously $x=0$ means that the whole population of players is playing $L$ and $x=1$ means that all the agents are playing $R$. This is the basic model of interaction that Schelling (1973) introduces in the first pages to define the uniform multi-person prisoner's dilemma. Part of the analysis in the rest of the paper is based on considerations on the payoff functions.

Even if no dynamic models are explicitly analyzed by Schelling, a dynamic adjustment is implicitly ${ }^{1}$ assumed in order to both analyze the time evolution of the fraction $x$, and the stability of the equilibrium points. The basic assumption of such dynamic adjustment is the following:

Assumption. $x$ will increase whenever $R(x)>L(x)$ whereas it will decrease when the opposite inequality holds.

This assumption, together with the constraint $x \in[0,1]$, implies that equilibria are located either in the points $x=x^{*}$ such that $R\left(x^{*}\right)=L\left(x^{*}\right)$ or, in $x=0$, provided that $R(0)<L(0)$ or, in $x=1$, provided that $R(1)>L(1)$. This again is consistent with the discussion on the illustrative curves reported in Figure 2 in Schelling (1973).

Since no explicit dynamic model is proposed, all the conclusions of Schelling (1973) are drawn either by qualitative arguments or by inspection of graphical representations of the payoff functions (see, e.g., the discussion on pp. 387-390 and the comments about Figures 69 in the original paper). Instead, we introduce an explicit process of repeated binary choices where a fixed number of agents update their binary choice at each time period $t=0,1,2, \ldots$, and let $x_{t}$ be the number of players playing strategy $R$ at time $t$. We assume that at time $t+1, x_{t}$ becomes common knowledge; hence each agent is able to compute (or observe) payoffs $L\left(x_{t}\right)$ and $R\left(x_{t}\right)$. Finally we assume that agents are homogeneous and myopic, that is, each is interested in increasing its own next period payoff. The discrete-time model we propose is consistent with the assumptions of Schelling: we assume that if at time $t x_{t}$ players are playing strategy $R$ and $R\left(x_{t}\right)>L\left(x_{t}\right)$, then a fraction of the $\left(1-x_{t}\right)$ agents that are playing $L$ will switch to strategy $R$ in the following turn; analogously, if $R\left(x_{t}\right)<L\left(x_{t}\right)$ then a fraction of the $x_{t}$ players that are playing $R$ will switch to strategy

\footnotetext{
${ }^{1}$ See in Schelling (1973) the discussion on the differential payoff on page 391 and the discussion about coalitions starting on page 393 .
} 
$L$. In other words, at any time period $t$ agents decide their action for period $t+1$ comparing $L\left(x_{t}\right)$ and $R\left(x_{t}\right)$ according to

$$
x_{t+1}=f\left(x_{t}\right)= \begin{cases}x_{t}+\delta_{R} g\left[\lambda\left(R\left(x_{t}\right)-L\left(x_{t}\right)\right)\right]\left(1-x_{t}\right) & \text { if } R\left(x_{t}\right) \geq L\left(x_{t}\right) \\ x_{t}-\delta_{L} g\left[\lambda\left(L\left(x_{t}\right)-R\left(x_{t}\right)\right)\right] x_{t} & \text { if } R\left(x_{t}\right)<L\left(x_{t}\right)\end{cases}
$$

where $g: \mathbb{R}_{+} \rightarrow[0,1]$ is a continuos and increasing function, $\delta_{R}, \delta_{L} \in$ $[0,1]$ and $\lambda$ is a positive real number. Function $g$ modulates how the fraction of switching agents depends on the difference between the previous turn payoffs; it must hold $g(0)=0$ and $\lim _{z \rightarrow \infty} g(z)=1$. Parameters $\delta_{R}$ and $\delta_{L}$ represent how many agents may switch to $R$ and $L$, respectively; when $\delta_{R}=\delta_{L}$, there are no differences in the propensity to switch to either strategy. Finally, parameter $\lambda$ represents the switching intensity (or speed of reaction) of agents as a consequence of the difference between payoffs. In other words, decreasing values of $\lambda$ imply more inertia, that is, anchoring attitude, of the actors involved.

Notice that if both the payoff functions are continuous, then the map $f$ also is continuous in the whole interval [0,1], and its graph is contained in the strip bounded by two lines, being $\left(1-\delta_{L}\right) x \leq f(x) \leq$ $\left(1-\delta_{R}\right) x+\delta_{R}$. However, even if $L(x)$ and $R(x)$ are smooth functions, the map $f$ in general is not smooth in the considered interval, since $f$ is not differentiable where the payoff functions intersect. When studying the dynamics, this does not allow us to use the usual approach which relies on the first derivative value in the equilibrium point. As a consequence an approach based on the left and right side slopes in the neighborhood of any interior fixed point is needed.

Finally, an interesting limiting case is obtained as $\lambda \rightarrow+\infty$. This is equivalent to consider $g(\cdot)=1$; as a consequence the switching rate only depends on the sign of the difference between payoffs, no matter how much they differ. In this case the dynamical system assumes the following form

$$
x_{t+1}=f_{\infty}\left(x_{t}\right)= \begin{cases}\left(1-\delta_{R}\right) x_{t}+\delta_{R} & \text { if } L\left(x_{t}\right)<R\left(x_{t}\right) \\ x_{t} & \text { if } L\left(x_{t}\right)=R\left(x_{t}\right) \\ \left(1-\delta_{L}\right) x_{t} & \text { if } L\left(x_{t}\right)>R\left(x_{t}\right)\end{cases}
$$

This limiting situation is quite extreme and somewhat unrealistic since it describes a situation where a finite fraction of agents, $\delta_{R}$ and $\delta_{L}$, respectively, switch their strategies even when the difference between payoffs is extremely small. In addition, from a dynamic point of view, the map is not suitable to represent real time 
patterns as internal equilibria, which are defined by the equation $L(x)=R(x)$, represent points of discontinuity. However, as we shall see, the global properties of $f_{\infty}\left(x_{t}\right)$ may give some insight into both the global properties of the smooth map (2) and the role of the parameters $\delta_{R}$ and $\delta_{L}$.

\section{PAYOFF FUNCTIONS WITH ONE INTERSECTION}

In this section we consider payoff functions $R(x)$ and $L(x)$ with one and only one internal intersection; that is, there exists a unique point $x^{*} \in(0,1)$ such that $R\left(x^{*}\right)=L\left(x^{*}\right)$. We consider first the case where the strategy $R$ is preferred at the right of $x^{*}$ and $L$ is preferred at the left, as in Figure 1a. This situation is discussed in Schelling (1973), where a vivid example is also provided (pp. 382, 384), by assuming that $L$ stands for carrying a visible weapon and $R$ for going unarmed. One may prefer to be armed if everybody else is but not if the rest are not (individuals may also be nations; see, e.g., Appendix A in Schelling, 1960), and the visibility of weapons is an essential assumption for the model as it is proposed. According to the relative position of the two payoff curves, in Schelling (1973, p. 403) the author concludes that there exist two stable equilibria, namely $x=0$ and $x=1$, where everybody is choosing $L$ and everybody is choosing

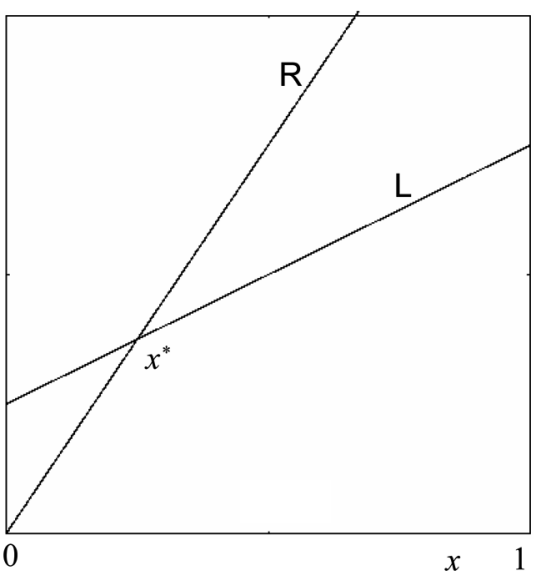

(a)

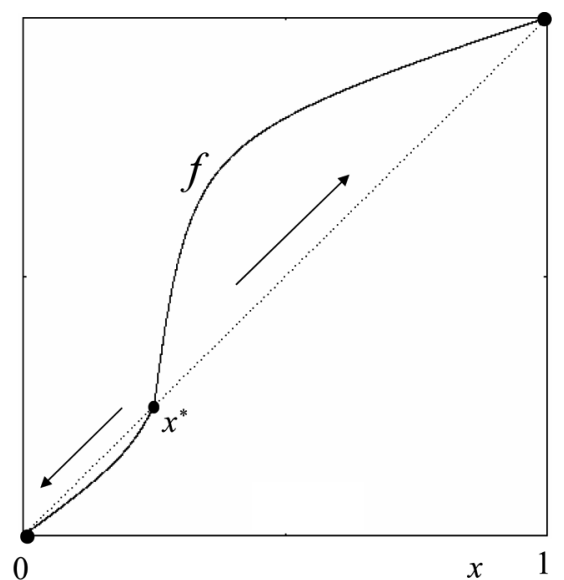

(b)

FIGURE 1 (a) Payoff functions $R(x)=1.5 x$ and $L(x)=0.25+0.5 x$. (b) Function $f$ obtained with the same payoff functions as in (a) and parameters $\delta_{L}=0.3, \delta_{R}=0.7, \lambda=20$ with switching function $g(\cdot)=\frac{2}{\pi} \arctan (\cdot)$. 
$R$, respectively, whereas the inner equilibrium $x^{*}$ is unstable. The reasons given by Schelling to prove these statements are based on the following arguments: at the equilibrium $x=0$, where everybody is choosing $L$, nobody is motivated to choose $R$ because $R(x)<L(x)$ in a right neighborhood of 0 , and analogously at $x=1$, where everybody is choosing $R$, nobody is motivated to choose $L$ being $L(x)<R(x)$ in a left neighborhood of 1 ; instead, starting from the inner equilibrium $x^{*}$, where both choices coexist, if $x$ is displaced in a right neighborhood of $x^{*}$ by an exogenous force, there $R(x)>L(x)$ and a further increase of $x$ will be generated by endogenous dynamics. If $x$ is displaced in a left neighborhood of $x^{*}$, where $R(x)<L(x)$, then a further decrease of $x$ will be observed.

We can confirm the results of Schelling's analysis by using the explicit dynamic model (1). In fact, it is easy to prove that $f(0)=0$ so that $x=0$ is a fixed point of (1); furthermore as $f^{\prime}(0)=$ $1-\delta_{L} g[\lambda(L(0)-R(0))]$, it holds $0<f^{\prime}(0)<1$ being $0 \leq \delta_{L} \leq 1$ and $0 \leq g(\cdot)<1$, as a consequence $x=0$ is a stable fixed point with monotonic convergence (see, e.g., Devaney, 1989; Robinson, 1999). The same holds for $x=1$, since $f(1)=1$ and $0<f^{\prime}(1)<1$. Finally consider $x^{*}$ such that $f\left(x^{*}\right)=x^{*}$; there the slopes of both left and right tangents, say $f_{-}^{\prime}\left(x^{*}\right)$ and $f_{+}^{\prime}\left(x^{*}\right)$ respectively, are greater than 1 as

$$
\begin{aligned}
& f_{-}^{\prime}\left(x^{*}\right)=1-\delta_{L}\left[\lambda g^{\prime}(0)\left(L^{\prime}\left(x^{*}\right)-R^{\prime}\left(x^{*}\right)\right)\right] x^{*}>1, \\
& f_{+}^{\prime}\left(x^{*}\right)=1+\delta_{R}\left[\lambda g^{\prime}(0)\left(R^{\prime}\left(x^{*}\right)-L^{\prime}\left(x^{*}\right)\right)\right]\left(1-x^{*}\right)>1
\end{aligned}
$$

since $L^{\prime}\left(x^{*}\right)<R^{\prime}\left(x^{*}\right)$.

If we consider the two payoff functions depicted in Figure 1a, with the particular choice $g(\cdot)=\frac{2}{\pi} \arctan (\cdot)$ we obtain the function $f$, depicted in Figure 1b. Observe that with any other function $g$ having the properties listed above, it is possible to find a map $f$ which is similar in shape. Furthermore, on the basis of continuity arguments, and without any assumption of differentiability for payoff functions $L$ and $R$, similar conclusions can be drawn.

These results can be summarized by the following:

Proposition 1. Assume that $R:[0,1] \rightarrow \mathbb{R}$ and $L:[0,1] \rightarrow \mathbb{R}$ are continuous functions such that

- $R(0)<L(0)$

- $R(1)>L(1)$

- there exists unique $x^{*} \in(0,1)$ such that $R\left(x^{*}\right)=L\left(x^{*}\right)$,

then dynamical system (1) has three fixed points, $x=0, x=x^{*}$ and $x=1$, where $x^{*}$ is unstable and constitutes the boundary that separates 
the basins of attraction of the stable fixed points 0 and 1. All the dynamics generated by (1) converge to one of the two stable fixed points monotonically, decreasing if $x_{0}<x^{*}$, increasing if $x_{0}>x^{*}$.

This result confirms the same conclusions that Schelling (1973, p. 403) deduced qualitatively by direct inspection of payoff curves. Furthermore, the previous result can be reformulated locally as follows:

Corollary 1. Assume that $R:[0,1] \rightarrow \mathbb{R}$ and $L:[0,1] \rightarrow \mathbb{R}$ are continuous functions and that there exists $x^{*} \in(0,1)$ and a neighborhood $U\left(x^{*}\right)$ such that

- $R\left(x^{*}\right)=L\left(x^{*}\right)$

- $R(x)<L(x)$ for all $x \in \mathscr{U}\left(x^{*}\right)$ such that $x<x^{*}$

- $R(x)>L(x)$ for all $x \in \mathscr{U}\left(x^{*}\right)$ such that $x>x^{*}$

then $x^{*}$ is a repulsive fixed points, for the dynamical system (1). Considering the restriction of any dynamics to $U\left(x^{*}\right)$ the repulsiveness of $x^{*}$ is monotonic: decreasing if $x_{0}<x^{*}$, and increasing if $x_{0}>x^{*}$.

By contrast, the situation is quite different when the payoff functions are switched, that is, $R(0)>L(0)$ and $R(1)<L(1)$, so that $R$ is preferred at the left of the unique intersection $x^{*}$ and $L$ is preferred at the right (see Fig. 2a). In this case we have a unique equilibrium, given by the interior fixed point $x^{*}$.

Schelling (1973) describes this case as well and provides some real-life examples of collective binary choices with this kind of payoff functions. Among these examples one concerns the binary choice about whether using the car or not, depending to the traffic congestion. Let $R$ represent the strategy "staying at home" and $L$ "using the car." If many individuals choose $L$ (i.e., $x$ is small) then $R$ is preferred because of traffic congestion, whereas if many choose $R$ (i.e., $x$ is large) then $L$ is preferred as the roads are empty. This situation can be represented with payoff functions as those depicted in Figure 2a. Schelling (1973, p. 401), gives a qualitative analysis of this scenario and classifies it as being characterized by global stability of the unique equilibrium point. Using the words of Schelling (1973), "[i]f we suppose any kind of damped adjustment, we have a stable equilibrium at the intersection" (p. 401). His argument is based on the fact that $R(x)>L(x)$ on the left of $x^{*}$ (hence increasing $x$ whenever $x<x^{*}$ ) and $R(x)<L(x)$ on the right of $x^{*}$ (hence decreasing $x$ whenever $\left.x>x^{*}\right)$. While this statement of global stability is true when assuming a continuous time scale, in our case we observe a 


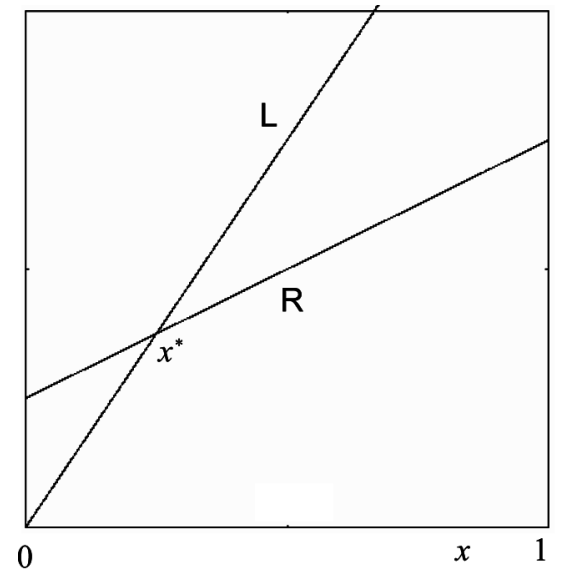

(a)

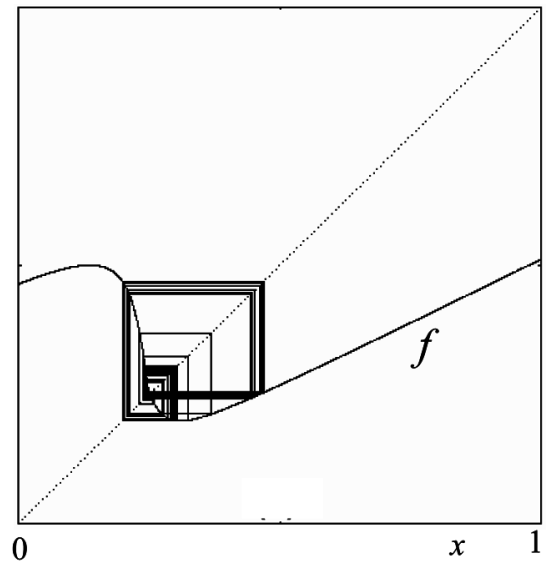

(b)

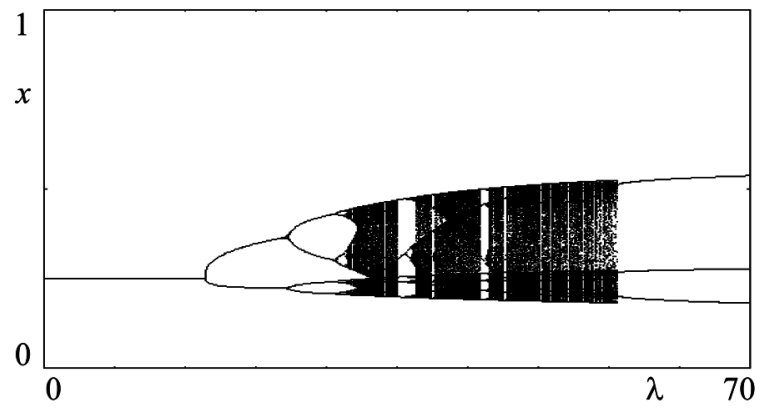

(c)

FIGURE 2 (a) Payoff functions $L(x)=1.5 x, R(x)=0.25+0.5 x$. (b) Function $f$ obtained with $g(\cdot)=\frac{2}{\pi} \arctan (\cdot)$, the same payoffs as in (a) and parameters $\delta_{L}=\delta_{R}=0.5, \lambda=35$. The interior equilibrium is unstable and the generic trajectory converges to the attractor shown around $x^{*}$. (c) Bifurcation diagram obtained with the same values of parameters $\delta$ and payoff functions as in (b) and bifurcation parameter $\lambda \in(0,70)$.

different behavior since we consider discrete time. In fact, in our discrete-time model we can observe oscillations of $x_{t}$, an occurrence which is a priori excluded in the continuous time dynamics.

By contrast, when considering the discrete-time dynamic model (1) we realize that the slope of the function $f$ at the steady state may be positive or negative. More precisely, since $L^{\prime}\left(x^{*}\right)>R^{\prime}\left(x^{*}\right)$, both the the left and right tangents $f_{-}^{\prime}\left(x^{*}\right)$ and $f_{+}^{\prime}\left(x^{*}\right)$ are less than 1:

$$
f_{-}^{\prime}\left(x^{*}\right)=1+\delta_{R}\left[\lambda g^{\prime}(0)\left(R^{\prime}\left(x^{*}\right)-L^{\prime}\left(x^{*}\right)\right)\right]\left(1-x^{*}\right)<1
$$


and

$$
f_{+}^{\prime}\left(x^{*}\right)=1-\delta_{L}\left[\lambda g^{\prime}(0)\left(L^{\prime}\left(x^{*}\right)-R^{\prime}\left(x^{*}\right)\right)\right] x^{*}<1
$$

Hence $x^{*}$ is stable as far as $f_{-}^{\prime}\left(x^{*}\right)>-1$ and $f_{+}^{\prime}\left(x^{*}\right)>-1$, and it may becomes unstable ${ }^{2}$ when at least one of these slopes decrease below -1 (see, e.g., Devaney, 1989; Robinson, 1999).

This last situation may occur, for example, when considering $L(x)=1.5 x, R(x)=0.25+0.5 x, g(\cdot)=\frac{2}{\pi} \arctan (\cdot), \delta_{L}=\delta_{R}=0.5, \lambda=35$, as shown in Figure 2b. In this case, $x$ increases in the right neighborhood of 0 and decreases in the left neighborhood of 1 ; nevertheless, the unique equilibrium $x^{*}$ may be unstable, and persistent oscillations, periodic or chaotic, may be observed around it. We can summarize the wide spectrum of asymptotic dynamic behaviors by the bifurcation diagram depicted in Figure 2c, which is obtained with the same values of parameters $\delta$ and payoff functions as in Figure $2 \mathrm{~b}$ and by considering the parameter $\lambda$ that varies in the range $(0,70)$. The complex behaviors depicted in Figure $2 \mathrm{c}$ are an example of the chaotic behavior which characterizes many social systems; for a discussion about the chaos and complexity in sociology the reader may refer to Eve, Horsfall, and Lee (1997). However, as shown in Figure 2c, for high values of the parameter $\lambda$, the asymptotic dynamics settle on a given periodic cycle (of period 3 in this case) according to the values of the parameters $\delta_{L}$ and $\delta_{R}$. This can be easily forecasted from the study of the limiting map (2), as shown in Section 4.

The occurrence of oscillations is typical of a discrete-time process and is caused when individual players overshoot or overreact. For example, in the model of binary choice in car usage described above, overshooting occurs for sufficiently large values of $\lambda$ (high speed of reaction). This means that whenever traffic congestion is reported, on the following day most people will stay at home; when no traffic congestion is reported most all of the people will use their cars. This kind of reactions generates a typical oscillatory time pattern which is a common situation observed in everyday life. This sort of realistic situation would be completely ruled out when adopting a continuous time one-dimensional dynamic model.

Obviously, even in a discrete time setting, oscillations may be avoided or at least damped. In this case, if a sufficient degree of

\footnotetext{
${ }^{2}$ As the function $f$ is not differentiable in its fixed points, when the derivative is below -1 only on one side, the equilibrium may be stable or unstable according to the global properties of the function, in the sense that the equilibrium may be locally repelling from one side but globally asymptotically stable if the long run dynamics is governed by the stable branch.
} 
inertia is present, that is, low values of the speed of reaction $\lambda$, the convergence to the equilibrium $x^{*}$ is achieved. In the same example this means that people may learn to "discount the (traffic) warning" and consequently may exhibit a "low propensity to switch." Also, reducing the time lags between traffic observations and decisions may be effective in reducing oscillations; this could be obtained by subscribing a service that keeps people currently informed so that they can more frequently revise their decisions. All these factors may help to reach the unique equilibrium, that is, giving it stability and avoiding overshooting effects.

However, it is plain that several of the examples proposed in the literature, and in particular in Schelling's paper, are characterized by decisions that cannot be continuously revised, and lags between observations and decisions are often finite. As a consequence, decision processes typically occur in a discrete time setting. This implies, as is well known in general, a much richer spectrum of dynamic behaviors than the one observed in continuous time one-dimensional models. The reader may also refer to Schelling (1978, ch. 3), for several others qualitative descriptions of overshooting and cyclic phenomena in social systems.

The results of our discussion can be summarized in

Proposition 2. If $R:[0,1] \rightarrow \mathbb{R}$ and $L:[0,1] \rightarrow \mathbb{R}$ are continuous functions such that

- $R(0)>L(0)$

- $R(1)<L(1)$

- there exists unique $x^{*} \in(0,1)$ such that $R\left(x^{*}\right)=L\left(x^{*}\right)$,

then the dynamical system (1) has only one fixed point at $x=x^{*}$, which is stable if $f_{-}^{\prime}\left(x^{*}\right)>-1$ and $f_{+}^{\prime}\left(x^{*}\right)>-1$, and may be unstable if at least one of these two slopes is smaller than -1 . Both slopes decrease as $\lambda$ or $\delta_{L}$ or $\delta_{R}$ increase, i.e., if the propensity to switch to the opposite choice increases.

The previous result can be reformulated locally as well.

Corollary 2. Assume that $R:[0,1] \rightarrow \mathbb{R}$ and $L:[0,1] \rightarrow \mathbb{R}$ are continuous functions and that there exists $x^{*} \in(0,1)$ and $a$ neighborhood $U\left(x^{*}\right)$ such that

- $R\left(x^{*}\right)=L\left(x^{*}\right)$

- $R(x)>L(x)$ for all $x \in \mathscr{U}\left(x^{*}\right)$ such that $x<x^{*}$

- $R(x)<L(x)$ for all $x \in U\left(x^{*}\right)$ such that $x>x^{*}$ 


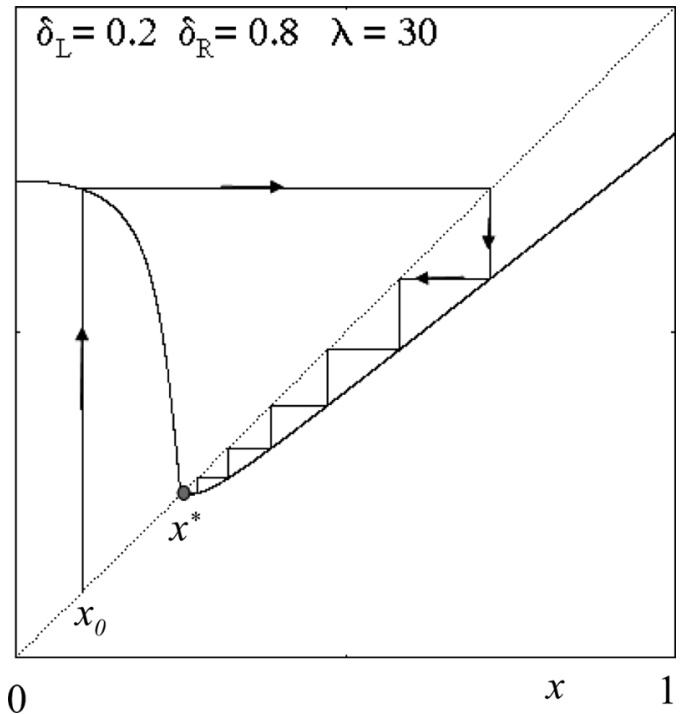

FIGURE 3 An exemplary case where $f_{-}^{\prime}\left(x^{*}\right)<-1$ and $0<f_{+}^{\prime}\left(x^{*}\right)<1$. Even if the fixed point $x^{*}$ is locally repelling on the left, it is asymptotically stable because all the trajectories converge to it in the long run.

then $x^{*}$ is a fixed points, which is stable if $-1<f_{-}^{\prime}\left(x^{*}\right)$ and $-1<f_{+}^{\prime}\left(x^{*}\right)$, and may be unstable if at least one of these two slopes is smaller than -1 .

We stress again that, due to the presence of a kink (point where the map $f$ is not smooth) at $x=x^{*}$, when $f_{-}^{\prime}\left(x^{*}\right)<-1$ and $0<f_{+}^{\prime}\left(x^{*}\right)<1$ even if the fixed point is locally repelling from one side, that is, the derivative is below -1 only on one side, the equilibrium may be stable if the dynamics are ultimately governed by the attracting branch, as qualitatively shown in Figure 3.

\section{PAYOFF FUNCTIONS WITH TWO INTERSECTION POINTS}

The existence of nonmonotonic payoff functions may lead to the existence of more than one intersection, that is, two or more interior equilibria exist. This may imply the existence of several stable equilibria, each with its own basin of attraction. An example that exhibits the existence of two interior equilibria is given by the recent threats posed by the resistance to antibiotics reported in the medical literature (see, e.g., Seppala et al., 1992). Consider the introduction 
of new antibiotic in a population and assume individuals are facing the binary choice to adopt or not to adopt the antibiotic. On one side, if the fraction of population which potentially could choose the new antibiotic is small, then the production would be almost null and any single individual will not find economically viable to use it due to the high price. On the other side, if the fraction of population using that antibiotic is very high, then it may become less effective due to the appearance of bacteria resistant to that kind of antibiotic. As a consequence, only for intermediate levels of diffusion adopting the antibiotic would be the best choice. This will lead to the creation of a stable equilibrium that gives an intermediate level of antibiotic diffusion. However, if some marketing policy will force an excessive spread of the antibiotic, it will become so ineffective that nobody will choose it in the future, that is, an irreversible transition toward the disappearance of the antibiotic occurs (see, e.g., Leclercq, 2002). This is just an example; however, it is not difficult to find other situations where an excessive diffusion of a given collective choice induces an extreme form of over-reaction leading to fast transition to the opposite choice. While this kind of occurrence is completely ruled out in Schelling (1973), it may be explained parsimoniously by our model. In fact, with our modellization it is possible to explain these kind of phenomena, in terms of basins of attraction of competing equilibria and global bifurcations that create non connected basins. They are equivalent to the creation of multiple thresholds that mark different equilibrium selections in the long run. In the antibiotics example and, more generally, in the study of the evolution of social systems, the problem of path dependence, is an important issue. Specifically, the problem of path dependence concerns, which equilibrium will prevail in the long run, how this is influenced by changes of the initial conditions, as well as displacements of initial conditions due to historical accidents, and, finally, also the irreversible departure from an equilibrium similar to the one described above.

However, this problem requires a deeper analysis of the mathematical properties of the dynamic model. Indeed, the results of Section 3 have been obtained through a local stability analysis, as we considered the linear approximation of the iterated map in the neighborhood of the equilibria, that is, the slopes of the graph of the map $f$ at (or near) the fixed points. Instead, to understand some global dynamic phenomena observed in the iteration of a nonlinear dynamical system, local stability analysis alone is not sufficient and may even be misleading. As a consequence, a global analysis is required; this approach consist of the localization of relative maximum and minimum values of the iterated map and 
their images (also called critical points; see Gumowski and Mira, 1980; Mira, Gardini, Barugola, and Cathala, 1996) as well as their contacts with unstable fixed points or cycles. These contacts may lead to interesting (and sometimes counterintuitive) qualitative changes, also called global bifurcations, in the topological structure of the attracting sets and their basins of attraction. Such changes can be easily observed when non monotonic payoff functions are considered, which may be a quite common situation (on this point, see also Granovetter, 1978).

In order to show what type of dynamic scenarios one may expect in such situations, and how the global bifurcations involved can explain some observed phenomena, we propose a specific example, where $L(x)=\frac{1}{2} x$ and $R(x)=-8 x^{2}+12 x-4$ (see Fig. 4a). We indicate $x_{1}^{*}<x_{2}^{*}$ the two distinct points where $L(\cdot)$ and $R(\cdot)$ intersect; in this case there exist three equilibria: $x=0, x=x_{1}^{*}$ and $x=x_{2}^{*}$. In the same figure the map $f$, obtained with the usual choice $g(\cdot)=\frac{2}{\pi} \arctan (\cdot)$, is also reported. With reference to the example of antibiotic diffusion described above, $x_{1}^{*}$ represents the lower threshold below which it choosing the given antibiotic is difficult due to lack of demand, $x_{2}^{*}$ represents the upper equilibrium above which the side effect of bacteria resistance to a too diffused antibiotic becomes important.

Schelling (1973, p. 414) and Granovetter (1978, p. 1438) analyze nonmonotonic payoff curves that assume such a configuration, and

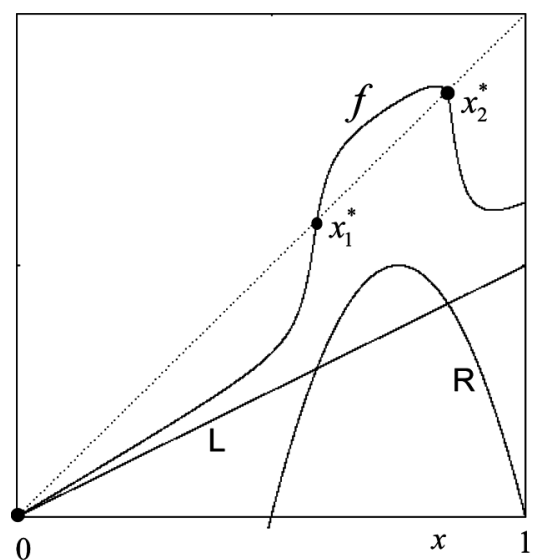

(a)

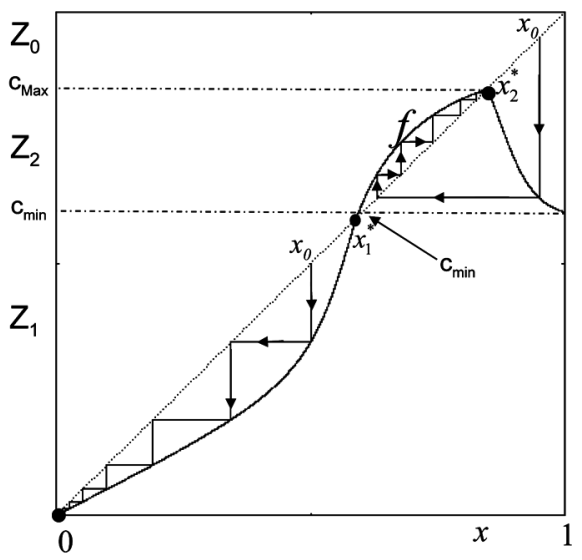

(b)

FIGURE 4 (a) Payoff functions $L(x)=\frac{1}{2} x$ and $R(x)=-8 x^{2}+12 x-4$ together with the map $f$ obtained with $g(\cdot)=\frac{2}{\pi} \arctan (\cdot), \delta_{R}=\delta_{L}=0.5, \lambda=6$. (b) Two trajectories starting from initial condition $x_{0}=0.95$ and $x_{0}=0.5$. 
relate them to several real life situations. Schelling (1973, p. 414) also describes an example concerning the binary choice of using or not insecticide in the garden, in the presence of other gardens around. Let $R$ represent "use insecticide" choice and $L$ not using it. If nobody around use insecticide (low value of $x$ ) then the effectiveness local application of insecticide is very low because there are many bugs coming from gardens around. Hence one may decide that it is not convenient spending money to buy it. On the other side, if all neighbors use insecticide (high values of $x$ ), then there are not enough bugs around to warrant spending money to spray insecticide locally. As a consequence, only in the presence of moderate usage by others (i.e., intermediate values of $x$ ) it becomes cost-effective to apply insecticide locally. The payoff curves shown by Schelling (1973, p. 414) to describe this situation are similar to the ones shown in Figure 4.

Also, Granovetter (1978, p. 1439), provides a vivid example related to the presence of two threshold values, concerning the decision of going or not to a restaurant: if the place is nearly empty it is probably a bad sign and one would try another place, but if the restaurant is so crowded that the waiting time is unbearable it will also lead to choose another place.

Another meaningful example may be found in economics when one considers the trade-off between research and development (R\&D) expenditures and knowledge spillovers among firms producing similar goods in an industrial district (see, e.g., Bischi and Lamantia, 2002; Bischi, Dawid, and Kopel, 2003a, 2003b). Research and development investments in order to produce and sell a given product may be quite inefficient if nobody else produces the same good because no available information exist. On the other side, if, in an industrial district, too many firms produce the same good and invest in $R \& D$, then a firm can take advantage, for free, of the competitors' R\&D results, due to the difficulties to protect intellectual properties or to avoid the movements of skilled workers among competing firms.

These are just a few among several interesting real-life examples that can be described by nonmonotonic payoff functions with two internal intersections; consequently a detailed study of the dynamic scenarios generated by such kind of payoff functions may be interesting in terms of both results and predictions. Moreover, as it will become clearer in the following, the global dynamics effects of the dynamical systems we are considering are interesting since they may explain in a parsimonious way, just changing the time scale of Schelling's model, phenomena that are common in social systems. In fact, studying the bifurcations and global dynamic properties, we can observe some typical non linear effects that lead to the creation 
of particular structures of the basins of attraction that help to explain the extreme forms of path dependence and irreversible overshooting described so far.

For example, consider the same payoff functions as in Figure 4a; fix parameters values as $\delta_{R}=\delta_{L}=0.5, \lambda=6$, then while $x=0$ and $x=x_{2}^{*}$ are stable, fixed point $x_{1}^{*}$ is unstable and is the unique separator of the two basins of attraction (see Fig. 4b). This map is such that, for all initial conditions $x_{0}>x_{1}^{*}$, the corresponding time evolutions (or trajectories) converge ${ }^{3}$ to the upper equilibrium $x_{2}^{*}$, and for all the initial conditions $x_{0}<x_{1}^{*}$ the trajectories converge to $x=0$. This is illustrated in Figure $4 \mathrm{~b}$ where two trajectories, starting from initial conditions $x_{0}=0.95$ and $x_{0}=0.5$ respectively, are shown. As a consequence, the unstable fixed point $x_{1}^{*}$ is the watershed that separates the two basins of attraction.

The local stability results we presented in Section 3 can be applied: Corollary 1 to $x_{1}^{*}$ and Corollary 2 to $x_{2}^{*}$. In particular, we stress that as in the neighborhood of $x_{2}^{*}$ slopes can be negative an oscillating behavior may occur; these oscillations may be either damped when $x_{2}^{*}$ is stable, periodic or chaotic when $x_{2}^{*}$ is unstable. The latter situations can be easily obtained when the speed of reaction $\lambda$ is sufficiently large, provided that $\delta_{L}$ and $\delta_{R}$ are not too small. In other words, a periodic or a chaotic attractor, say $A\left(x_{2}^{*}\right)$, may exist around $x_{2}^{*}$. However, all the arguments given above about the basins of attraction, as well as the considerations on basins' bifurcations we shall make in the following, remain the same when applied to the basin $\mathfrak{B}\left(A\left(x_{2}^{*}\right)\right)$ of the more complex attractor $A\left(x_{2}^{*}\right)$.

However, it is even more interesting that with no change in the local stability properties of the three equilibria, a global (or contact) bifurcation may occur, if some parameters vary opportunely, then the topological structure of the basins of attraction changes, as they are transformed into nonconnected sets. Such a structure of the basins can only be observed in discrete dynamical systems represented by the iteration of noninvertible maps (see, e.g., Mira et al., 1996; Agliari, Bischi, and Gardini, 2002), and the transition from simple connected basins (separated by an unstable fixed point) to nonconnected basins is caused by a contact between the unstable fixed point and the relative minimum value of the function $f$, say $c_{\min }$, located on the right of $x_{2}^{*}$.

The main point is that the map $f$ is noninvertible: there exist at least a pair of distinct points that are mapped into the same point,

\footnotetext{
${ }^{3}$ Notice that also in this case $x_{2}^{*}$ is globally asymptotically stable even if $f_{+}^{\prime}\left(x_{2}^{*}\right)<-1$.
} 
that is, distinct points with the same image, say $x_{1} \neq x_{2}$ such that $f\left(x_{1}\right)=f\left(x_{2}\right)$. This can be equivalently stated by saying that, in the range of the map $f$, there exist at least one point with several distinct preimages; the consequence is that the range of the map can be divided into different portions, or zones, characterized by a different number of preimages. Following the notation introduced in Mira et al. (1996), we denote by $Z_{k}$ the subset of points, in the range of $f$, that have $k$ preimages.

In the particular case of the map $f$ represented in Figure $4 \mathrm{~b}$, $Z_{1}=\left[0, c_{\min }\right), Z_{2}=\left(c_{\min }, c_{\mathrm{Max}}\right]$, and $Z_{0}=\left(c_{\mathrm{Max}}, 1\right)$, where $c_{\min }$ and $c_{\mathrm{Max}}$, respectively, represent the relative minimum and maximum values. In addition, as it concerns the unstable fixed point $x_{1}^{*}$ (located on the boundary that separates the two basins) it can be observed that $x_{1}^{*}<c_{\min }$; as a consequence $x_{1}^{*} \in Z_{1}$ and the point itself is its unique preimage as in an invertible map. This is the reason why $x_{1}^{*}$ is the unique point that forms the boundary separating the two basins of attraction. In fact, when as the result of a parameter variation that is large enough, the minimum value $c_{\min }$ is shifted downwards, and we have $c_{\min }<x_{1}^{*}$. In this case, then $x_{1}^{*} \in Z_{2}$ and there exists one more preimage, say $x_{1}^{*(-1)}$ belonging to the basin boundary as well. Figure 5 , which is obtained with all the same parameters as Figure 4 but $\lambda=10$, illustrates this situation. As it can be observed in Figure 5a,

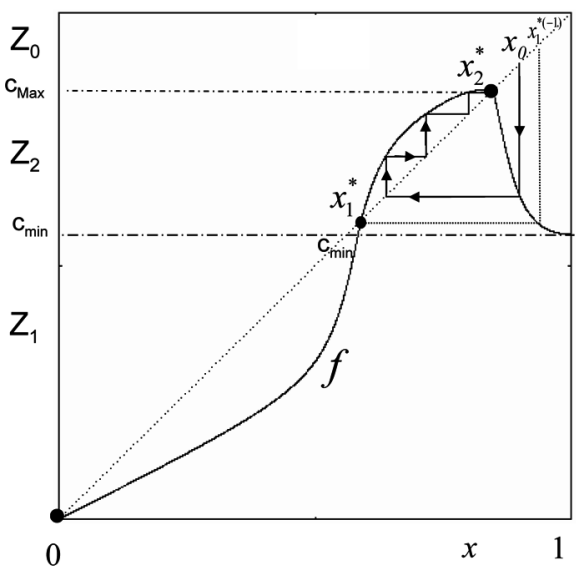

(a)

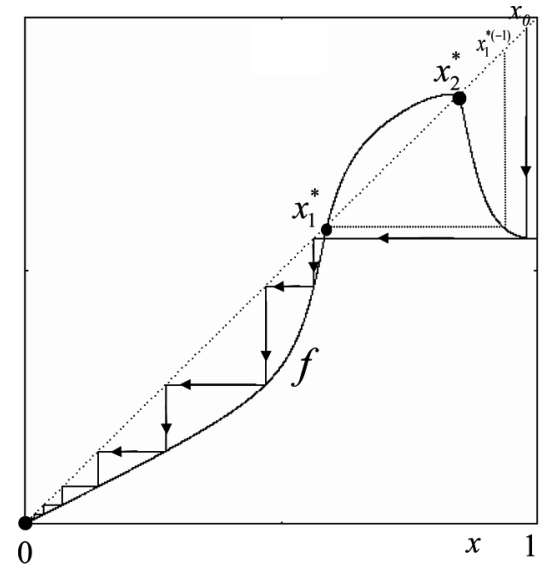

(b)

FIGURE 5 Map $f$ obtained with the same parameters as Figure 4 but $\lambda=10$. (a) The trajectory starting from $x_{0}=0.9$ converges to $x_{2}^{*}$. (b) The trajectory starting from $x_{0}=0.98$ converges to the fixed point $x=0$. 
while any initial condition $x_{0} \in\left(x_{1}^{*}, x_{1}^{*(-1)}\right)$ generates a trajectory that converges to $x_{2}^{*}$, on the contrary any initial condition $x_{0} \in\left(x_{1}^{*(-1)}, 1\right)$ is first mapped below $\left(0, x_{1}^{*}\right)$ and then converges to the fixed point $x=0$. In other words, the basin of the stable fixed point $x=0$ (everybody is choosing $L)$ is now $\mathfrak{B}(0)=\left(0, x_{1}^{*}\right) \cup\left(x_{1}^{*(-1)}, 1\right)$, that is, a nonconnected set, with the "hole" $\mathfrak{B}\left(x_{2}^{*}\right)=\left(x_{1}^{*}, x_{1}^{*(-1)}\right)$ "nested" inside. We recall that the widest component of the basin that contains the attractor is called immediate basin of the attractor.

To sum up, in this case there exists a value $\lambda$ such that the the topological structure of the basins exhibits a qualitative change; this value is characterized by the contact $c_{\min }=x_{1}^{*}$ between a critical point (relative minimum value) and an unstable fixed point. This global bifurcation leads to a counterintuitive behavior of the system. As the initial fraction of the populations of players choosing strategy $R$ (the initial condition $x_{0}$ ) increases from 0 to 1 , we first move from the basin of the lower equilibrium $x=0$ into the basin of the upper one $x_{2}^{*}$, to finally re-enter into the basin of attraction of 0 ; that is, while when many players initially choose $R$ the process will evolve towards a final equilibrium such that a large fraction of population chooses $R$, on the contrary when even more players initially choose $R$ then nobody will end up playing $R$ in the long run.

The situation may become even more involved when the position of the minimum is shifted horizontally so that global shape of the map $f$ implies a new zone $Z_{3}$. This is illustrated in Figure 6 , where parameters are $\delta_{R}=\delta_{L}=0.4, \lambda=40$. In this case $x_{1}^{*} \in Z_{3}$, actually as $\lambda$ increases a global bifurcation occurs: from $c_{\min }>x_{1}^{*}$ to $c_{\min }=x_{1}^{*}$ where the contact bifurcation occurs and, finally, $c_{\min }<x_{1}^{*}$ as in Figure 6. At this stage, there exist three distinct preimages of the boundary point $x_{1}^{*}: x_{1}^{*}$ itself and two more preimages denoted by $x_{1}^{*(-1), 1}$ and $x_{1}^{*(-1), 2}$ in Figure 6 . The result is that so that both basins consist of two disjoint portions: $\mathfrak{B}(0)=\left(0, x_{1}^{*}\right) \cup\left(x_{1}^{*(-1), 1}, x_{1}^{*(-1), 2}\right)$ and $\mathfrak{B}\left(A\left(x_{2}^{*}\right)\right)=$ $\left(x_{1}^{*}, x_{1}^{*(-1), 1}\right) \cup\left(x_{1}^{*(-1), 2}, 1\right)$.

In Figures $7 \mathrm{a}-\mathrm{c}$, three different trajectories can be observed; they are generated by initial conditions $x_{0}=0.8, x_{0}=0.91$ and $x_{0}=0.95$ respectively (of course, any $x_{0}<x_{1}^{*}$ generates a trajectory converging to 0 ). We also notice that, for this set of parameters, the larger fixed point $x_{2}^{*}$ is not stable, as around it there may exist a chaotic or highperiod periodic attractor. However, we can observe that the occurrence of the global bifurcation that changes the topological structure of the attractors is not influenced by the kind of coexisting attractors.

It is also worth noticing how the position of the maximum value $c_{\text {Max }}$ is important in bounding a trapping region which confines the 


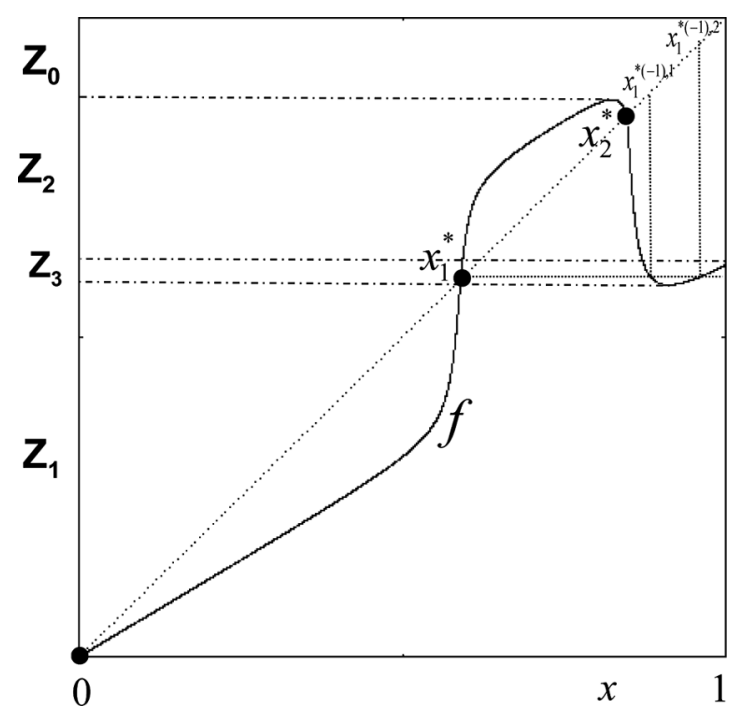

FIGURE 6 Map $f$ obtained with $\delta_{R}=\delta_{L}=0.4, \lambda=40$. In this case $x_{1}^{*} \in Z_{3}$.

asymptotic dynamics of any trajectories originating in a neighborhood of $x_{2}^{*}$. In fact, as can be seen in Figure 7, the chaotic attractor that exists around the fixed point $x_{2}^{*}$ is bounded above by the maximum value $c_{\text {Max }}$ and below by its image $f\left(c_{\text {Max }}\right)$. It is quite evident that if we iterate the map $f$ starting from any initial condition in the basin $\mathfrak{B}\left(A\left(x_{2}^{*}\right)\right)$, no values can be obtained above $c_{\text {Max }}$, and consequently no values can be mapped below its image $f\left(c_{\text {Max }}\right)$. In other words, interval formed by the critical point $c_{\text {Max }}$ and its image is trapping: any trajectory generated from an initial condition in $\mathfrak{B}\left(A\left(x_{2}^{*}\right)\right)$, after a finite number of iterations enters $\left[f\left(c_{\mathrm{Max}}\right), c_{\mathrm{Max}}\right]$, and then it never escapes out of it.

It is interesting to investigate whether, as $\lambda$ increases, the global bifurcation leading to the creation of nonconnected basins always occurs. In order to answer this question consider the piecewise linear (and piecewise continuous) map (2), which is the limiting case of map (1) obtained as $\lambda \rightarrow \infty$. This map, presents discontinuities at each point $x$ such that $R(x)=L(x)$, furthermore it consists of portions of the lower line $r_{L}(x)=\left(1-\delta_{L}\right) x$ (where $R(x)<L(x)$ ) and of the upper line $r_{R}(x)=\left(1-\delta_{R}\right) x+\delta_{R}$ (where $R(x)>L(x)$ ). Since $0 \leq g(\cdot) \leq 1$, for all $x \in[0,1]$ it results $r_{L}(x) \leq f(x) \leq r_{R}(x)$ (see Figure 8a). As a consequence, the study of the map (2) gives some general properties of the map $f$ independently of the value of $\lambda$, and allows us to emphasize 


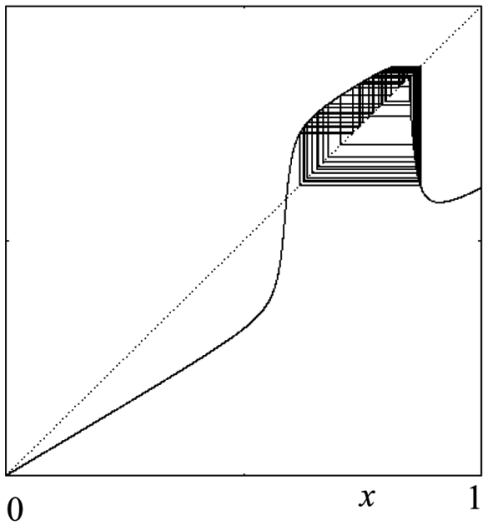

(a)

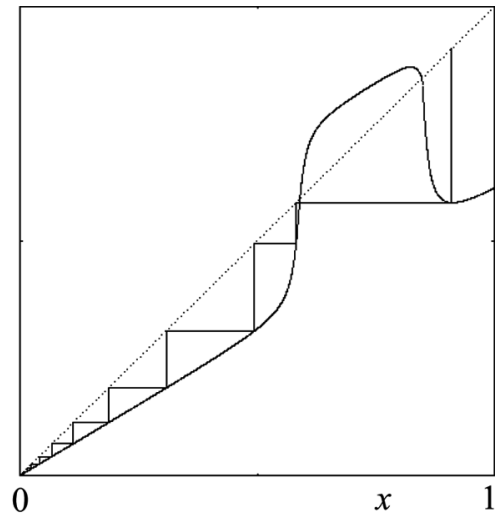

(b)

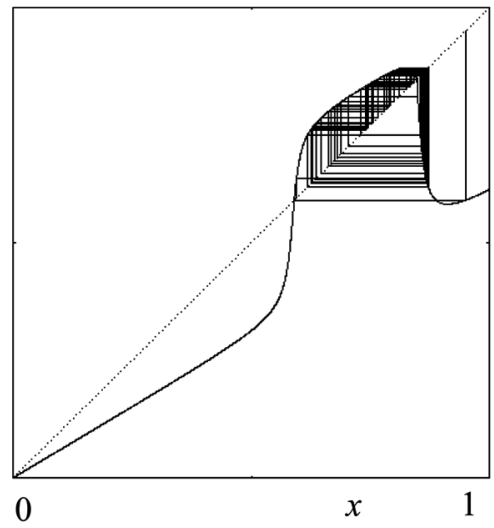

(c)

FIGURE 7 Three different trajectories obtained for the same map shown in Figure 6. (a) $x_{0}=0.8$. (b) $x_{0}=0.91$. (c) $x_{0}=0.95$.

the role of the parameters $\delta_{L}$ and $\delta_{R}$. For example, with the payoff functions we have been considering in this section, we have $c_{\mathrm{Max}} \leq$ $\left(1-\delta_{R}\right) x_{2}^{*}+\delta_{R}$ and $c_{\min } \geq\left(1-\delta_{L}\right) x_{2}^{*}$ (see Fig. 8b). As $\lambda$ increases, these inequalities allow us to find both a lower and an upper bound for the attractors $A\left(x_{2}^{*}\right)$. Moreover, from the second inequality we can deduce that if $\left(1-\delta_{L}\right) x_{2}^{*}>x_{1}^{*}$ then no global bifurcation leading to nonconnected basins may occur.

The study of the dynamic behaviors of the limiting map (2) can give some insight about the kind of dynamics we can expect as the parameter $\lambda$ increases. For example, assume the same two payoff 


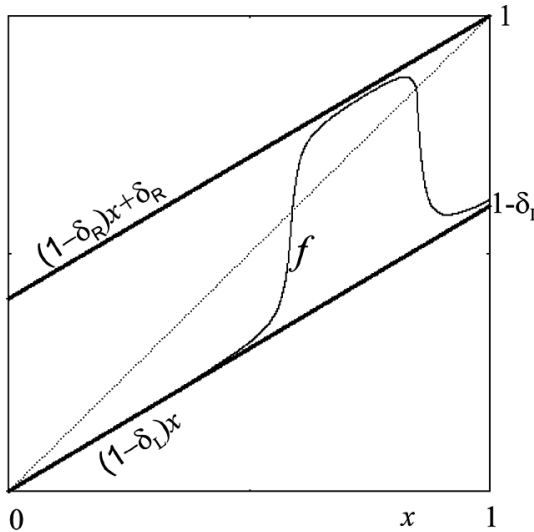

(a)

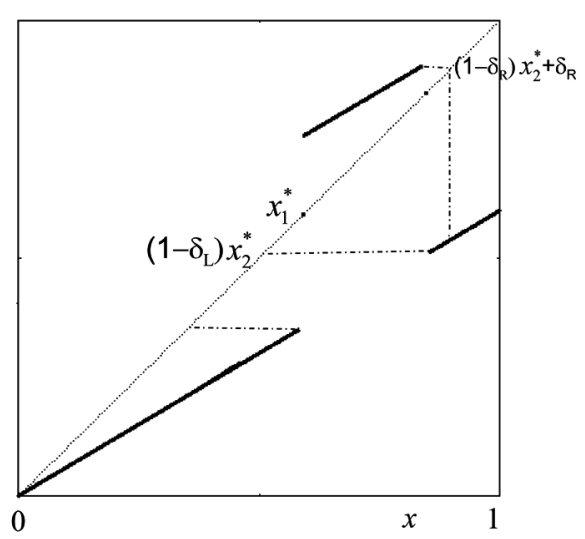

(b)

FIGURE 8 (a) The graph of the map $f$ is always included in the strip bounded by the two parallel lines $r_{L}(x)=\left(1-\delta_{L}\right) x$ and $r_{R}(x)=\left(1-\delta_{R}\right) x+\delta_{R}$. (b) The limiting map $f_{\infty}(x)$.

functions we have been considering so far. When $\delta_{R}=\delta_{L}=0.4$, for sufficiently large values of $\lambda$ the only long run dynamics we can observe is the convergence to the equilibrium $x=0$ (see Fig. 9a) whereas when assuming $\delta_{R}=0.4$ and $\delta_{L}=0.25$ then for sufficiently high values of $\lambda$ we have both the coexistence of the stable equilibrium $x=0$ and a stable cycle of period three around $x_{2}^{*}$, given by $A\left(x_{2}^{*}\right)=$ $\{0.877,0.657,0.794\}$ (Fig. 9b); finally observe that each basins of attraction is connected and that they are separated by $x_{1}^{*}$. Recall that assuming $\delta_{R} \neq \delta_{L}$ may be interpreted as the two choices being culturally nonequivalent.

The results discussed in this section may be summarized as follows:

Proposition 3. If $R:[0,1] \rightarrow \mathbb{R}$ and $L:[0,1] \rightarrow \mathbb{R}$ are continuous functions such that

- $R(0)<L(0)$

- $R(1)<L(1)$

- there exist two points $x_{1}^{*}<x_{2}^{*}$ both in $(0,1)$ such that $R\left(x_{i}^{*}\right)=L\left(x_{i}^{*}\right)$, $i=1,2$

then dynamical system (1) has three fixed points $x=0, x=x_{1}^{*}$, and $x=x_{2}^{*}$, where 0 is always stable, $x_{1}^{*}$ is always unstable, and $x_{2}^{*}$ may be stable or unstable. When $x_{2}^{*}$ is unstable, then a cyclic (periodic or 


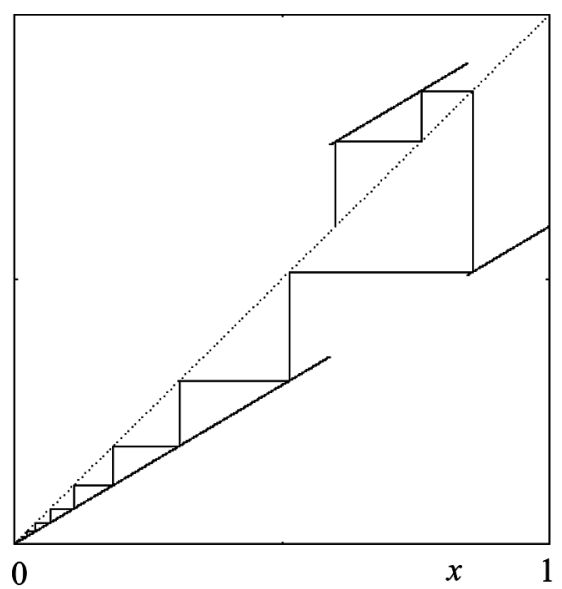

(a)

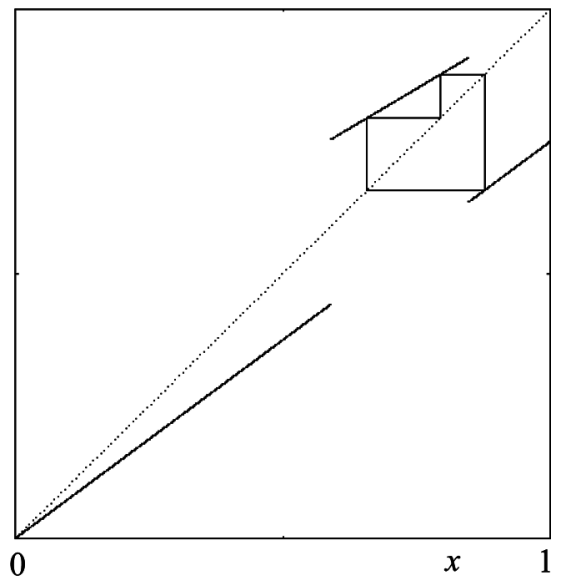

(b)

FIGURE 9 (a) Limiting map $f_{\infty}$ with $\delta_{R}=\delta_{L}=0.4$, all the trajectories converge to the equilibrium $x=0$; (b) With $\delta_{R}=0.4$ and $\delta_{L}=0.25$ then the iteration of $f_{\infty}$ shows coexistence of the stable equilibrium $x=0$ and a stable cycle of period three.

chaotic) attractor $A\left(x_{2}^{*}\right) \subseteq\left[f\left(c_{\text {Max }}\right), c_{\text {Max }}\right]$ exists around it and bounded inside the trapping set $\left[f\left(c_{\text {Max }}\right), c_{\text {Max }}\right]$, provided that $f\left(c_{\text {Max }}\right)>x_{1}^{*}$. The unstable fixed point $x_{1}^{*}$ is both the upper boundary of the immediate basin of the stable fixed point 0 , and the lower boundary of the immediate basin of $x_{2}^{*}$ (or $A\left(x_{2}^{*}\right)$ if it exists); furthermore, if $\left(1-\delta_{L}\right)$ $x_{2}^{*}>x_{1}^{*}$ then as $\lambda$ increases nonconnected portions of the basins are created.

The application of these results to the examples of insecticide or R\&D investments, described at the beginning of this section, is quite straightforward. Assume that both $\delta_{L}$ (the maximum fraction of agents switching to the strategy $L$ in one time period) and the speed of reaction $\lambda$ are sufficiently high. In this case an initial condition such that too many agents choose $R$ may lead to so many switches to strategy $L$ that the endogenous dynamics is no longer able to move back to $R$; the system remains trapped inside the everybody is choosing $L$ equilibrium, that is, $x=0$. By contrast, intermediate values of the fraction of agents initially choosing $R$ does not cause a so strong reaction against $R$; then either the collectivity settle to the upper equilibrium where the majority chooses $R$, or the number of agents choosing $R$ may oscillate forever around such 
equilibrium value, with average values approximately close to such upper equilibrium value.

\section{CONCLUSIONS}

In this article we considered an explicit discrete-time dynamic model to simulate an adaptive adjustment process of repeated binary choices with externalities, based on the qualitative properties described by Schelling (1973).

The choice of a discrete time scale, also suggested by Schelling (see footnote 9 in Schelling, 1960 and Schelling, 1978, pp. 85-87) and Granovetter (1978), allowed us to stress the occurrence of overshooting and cyclic phenomena in social systems. In particular, with monotonic payoff functions, the model proposed in this article allowed us to study the occurrence of oscillatory time series (periodic or chaotic).

The implicit dynamic adjustment proposed by Schelling fails in describing some important phenomena observed in real situations, such as oscillations caused by overshooting (or overreaction) of the actors involved in choices repeated over time, as well as problems of equilibrium selection when nonmonotonic payoff curves lead to the presence of several stable equilibria. In the latter case, the explicit dynamic model proposed in this paper acts as an equilibrium selection process and allows us to analyze structure of the basins of attraction, a problem not fully considered by Schelling. Our main result is given by the global analysis of the dynamic properties of the model that reveals the occurrence of a global bifurcation that causes the transition from connected to nonconnected basins of attraction. This implies that several basin boundaries (or thresholds, following the terminology of Granovetter, 1978) are suddenly created that may be seen as a possible mathematical description of an extreme form of path dependence, observed social systems, which is responsible of irreversible transitions from one equilibrium to another (and distant) one as final outcome.

Even if the results of this article are obtained for a particular dynamic model, proposed to simulate a repeated adaptive adjustment process, the mathematical methods used to obtain these results are quite general, and the conclusions obtained about the two kinds of complexity, related to complex attracting sets and complex structure of the basins of attraction, can be extended to general classes of discretetime adaptive models used to describe collective binary choices in social systems. The presence of overshooting and oscillatory behaviors should not be seen as an artificial effect or a distortion of reality 
due to discrete time scale. Instead, as stressed by Schelling (1978), overshooting and overreaction arise quite naturally in social systems due to emotional attitude, excess of prudence or lack of information. In fact, in our formal model overshooting appears as agents' propensity to switch to the opposite choice increases.

From a mathematical point of view, the kind of global dynamic analysis of the discrete time dynamical system we performed, based on a continuous dialogue among analytic, geometric and numerical methods, is based on the properties of noninvertible one-dimensional maps (see, e.g., Mira et al., 1996; Agliari et al., 2002). Such a global study of the dynamical system proposed here allow us to analyze, for given payoff functions, the quantitative and qualitative effects of the variations of the relevant parameters of the dynamic model on the topological structure of the basins of attraction, and we could obtain a delimitation of trapping intervals where the long-run behaviors of the model are confined. Finally, we gave some examples in the limit case of infinite switching propensity $\lambda \rightarrow+\infty$ and pointed to the consequences of relaxing the assumption of culturally equivalent choices $\delta_{R}=\delta_{L}$.

In future research we will further the analysis along two main avenues. On one hand, we are interested in analyzing the mathematical properties of piecewise maps; on the other hand, it would be quite interesting to analyze the consequences of heterogeneity of agents. This could be done either considering different individual payoffs or also assuming different social network structures in the agents population.

\section{ACKNOWLEDGMENTS}

The authors are grateful to two anonymous referees whose helpful suggestions greatly improved the quality of this article.

\section{REFERENCES}

Agliari, A., Bischi, G. I., \& Gardini, L. (2002). Some methods for the global analysis of dynamic games represented by noninvertible maps. In T. Puu \& I. Sushko (Eds.), Oligopoly Dynamics: Models and Tools (pp. 31-83). New York: Springer Verlag.

Bischi, G. I. \& Lamantia, F. (2002). Nonlinear duopoly games with positive cost externalities due to spillover effects. Chaos, Solitons \& Fractals, 13, 805-822.

Bischi, G. I., Dawid, H., \& Kopel, M. (2003a). Gaining the competitive edge using internal and external spillovers: a dynamic analysis. Journal of Economic Dynamics and Control, 27, 2171-2193.

Bischi, G. I., Dawid, H., \& Kopel, M. (2003b). Spillover effects and the evolution of firm clusters. Journal of Economic Behavior and Organization, 50, 47-75.

Braun. N. (1995). Individual thresholds and social diffusion. Rationality and Society, 7 , 167-182. 
Challet, D. \& Zhang, Y. C. (1997). Emergence of cooperation and organization in an evolutionary game. Physica A, 246-407.

Coleman, J. S. (1973). The Mathematics of Collective Action. Chicago, IL: Aldine.

Devaney, R. L. (1989). Chaotic Dynamical Systems (2nd ed.). Reading, MA: Perseus Books.

Durlauf, S. N. \& Young, P. (Eds.). (2001). Social Dynamics. Cambridge, MA: MIT Press.

Edling, C. R. (2002). Mathematics in sociology. Annual Review of Sociology, 28, 197-220.

Eve, R. A., Horsfall, S., \& Lee, M. E. (Eds.). (1997). Chaos, Complexity, and Sociology: Myths, Models, and Theories. London: Sage Publications.

Granovetter, M. (1978). Threshold models of collective behavior. The American Journal of Sociology, 83(6), 1420-1443.

Granovetter, M. \& Soong, R. (1983). Threshold models of diffusion and collective behavior. Journal of Mathematical Sociology, 9, 165-179.

Gumowski, I. \& Mira, C. (1980). Dynamique Chaotique. Toulose, France: Cepadues Editions.

Leclercq, R. (2002). Mechanisms of resistance to macrolides and lincosamides: nature of the resistance elements and their clinical implications. Clinical Infectious Diseases, $34,482-492$.

Mira, C., Gardini, L., Barugola, A., \& Cathala, J. C. (1996). Chaotic Dynamics in TwoDimensional Noninvertible Maps. Singapore: World Scientific.

Phan, D. \& Pajot, S. (2006). Complex behaviours in binary choice models with global or local social influence. In C. Bruun (Ed.), Lecture Notes in Economics and Mathematical Systems: Vol. 584. Advances in Artificial Economics-The Economy as a Complex Dynamic System (pp. 203-219). New York: Springer.

Robinson, C. (1999). Dynamical Systems (2nd ed.). Boca Raton, FL: CRC.

Schelling, T. C. (1960). The Strategy of Conflict. Cambridge, MA: Harvard University Press.

Schelling, T. C. (1973). Hockey helmets, concealed weapons, and daylight saving. Journal of Conflict Resolution, 17(3), 381-428.

Schelling, T. C. (1978). Micromotives and Macrobehavior. New York: W. W. Norton.

Seppala, H., Nissinen, A., Jarvinen, H., Huovinen, S., Henriksson, T., Herva, E., et al. (1992). Resistance to erythromycin in group. A streptococci. New England Journal of Medicine, 326, 292-297.

Sørensen, A. B. \& Sørensen, A. (1977). Mathematical Sociology: A Trend Report and a Bibliography. The Hague, The Netherlands: Mouton.

Young, H. P. (1998). Individual Strategy and Social Structure. Princeton, NJ: Princeton University Press. 